\title{
Des premiers pas de la thrombolyse préhospitalière à une prise en charge globale des syndromes coronariens aigus
}

\author{
B. Riou \\ (C) SFMU et Springer-Verlag France 2011
}

Ce numéro spécial des Annales françaises de médecine d'urgence élaboré avec l'aide des laboratoires AstraZeneca s'attache à décrire la prise en charge des syndromes coronariens aigus (SCA), notamment en ce qui concerne la prescription des médicaments destinés à accompagner la technique de reperfusion, quelle qu'elle soit, qui doit être mise en œuvre dans les délais les plus courts possibles [1]. Dans un premier article, Claret et al. [2] décrivent l'importance actuelle des filières de soins au cours de la prise en charge initiale des SCA en insistant sur le déclenchement de l'alerte devant une douleur thoracique, le rôle pivot des Samu-Centres 15 et l'importance de l'accès au défibrillateur pour diminuer la mortalité très précoce des SCA. Charpentier et Lauque [3] nous éclairent sur la stratégie diagnostique devant un SCA dans une approche moderne de la stratification du risque. Jacquin et al. [4] analysent les résultats de l'étude PLATO qui a comparé le ticagrelor et le clopidorel, deux inhibiteurs des récepteurs P2Y12 plaquettaires à l'ADP, dans les SCA [5]. Toutes ces études sur les médicaments antiagrégants plaquettaires à la phase précoce des SCA visent à diminuer les événements thrombotiques sans augmentation du risque hémorragique chez des patients souffrant d'un SCA et traités par angioplastie. Une mise au point sur la pharmacologie du ticagrelor sera par ailleurs publiée prochainement dans notre revue. Il s'agit d'un domaine extrêmement actif de la recherche pharmacologique, où la combinaison idéale n'est pas encore établie et où les avancées pharmacologiques sont importantes, rendant parfois caduques des notions pourtant établies de manière récente. C'est l'objectif du dernier article d'Assez et al. [6] que d'intégrer la place des inhibiteurs des récepteurs P2Y12 dans l'ensemble de notre arsenal thérapeutique pour la prise en charge en urgence des SCA.

\footnotetext{
B. Riou $(\square)$

Service d'accueil des urgences, groupe hospitalier Pitié-Salpêtrière,

Assistance publique-Hôpitaux de Paris,

Université Pierre-et-Marie-Curie-Paris-VI.

47-83, boulevard de l'Hôpital,

F-75651 Paris cedex 13, France

e-mail : bruno.riou@psl.aphp.fr
}

Il y a près de 25 ans, les résultats des premières thrombolyses préhospitalières dans le SCA pratiquées en France étaient publiés dans The Lancet [7]. De fait, il s'agissait d'une première mondiale, précédant de quelques mois seulement la publication de l'équipe israélienne de Tel Aviv [8]. Quand je me remémore ce qu'il faut bien qualifier de premiers pas de la médecine d'urgence dans l'univers de la cardiologie internationale, il convient de rappeler que les critiques étaient vives à l'époque, très peu d'équipes de cardiologie soutenant cette orientation. Même si elles étaient peu nombreuses à l'époque, les équipes de cardiologie françaises qui se sont investies dans cette coopération entre urgence et cardiologie ont écrit les premières pages d'une aventure médicale et scientifique assez exceptionnelle qui s'est traduite par des publications internationales remarquables et remarquées, des avancées importantes pour nos patients, et une habitude de collaboration entre nos deux disciplines qui ne s'est plus démentie depuis. Encore très récemment, et dans le cadre de grandes études multicentriques, les équipes de médecine d'urgence et de cardiologie ont montré leur capacité à créer la médecine de demain [9]. Dans la moitié des articles de ce numéro spécial, les urgentistes cosignent avec des cardiologues leur travail, démontrant l'intrication et la collaboration quotidienne entre ces deux disciplines.

À l'heure où les neurologues vasculaires souhaitent développer la prise en charge des urgences cérébrovasculaires, voulant ainsi suivre la trace exemplaire de la cardiologie interventionnelle française, il serait bon qu'ils se penchent sur ce qui a fait le succès de la filière de cardiologie interventionnelle française. Il n'y a pas eu besoin de créer des outils de régulation nouveaux, spécifiques et couteux, le succès est au contraire passé par l'utilisation d'un outil exceptionnel, polyvalent et implanté sur l'ensemble du territoire, les Samu-Centres 15. Il n'y a pas eu de tentative de mise sous tutelle des urgentistes, notamment pour la prescription des médicaments thrombolytiques. Il n'y a pas eu non plus de comportement condescendant quant à la capacité des urgentistes d'établir un diagnostic et un pronostic et de prendre des décisions. Au contraire, dans une démarche de partenariat entre les deux disciplines, il y a eu réflexion 
commune, établissements de protocoles communs, et finalement construction d'une filière très efficace au quotidien, permettant ainsi une collaboration redoutable d'efficacité dans la recherche clinique. Sur ce dernier point, la reconnaissance mutuelle est également passée par la cosignature des articles.

Puissent les autres disciplines tenir compte de cette histoire commune remarquable de près de 25 ans entre les urgences et la cardiologie, pour ne pas commettre des erreurs qui, in fine, retardent la mise en place d'un juste soin pour nos patients.

\section{Références}

1. Winjs W, Khol P, Danchin N, et al (2010) Guidelines on myocardial revascularisation. The task force on myocardial revascularization of the Eurpean Society of Cardiology (ESC) and the European Association for Cardio-Thoracic Surgery (EACTS) developed with the special contribution of the European Association of Percutaneous. Cardiovascular Interventions (EAPCI). Eur Heart J $31: 2501-55$
2. Claret B, Benezet JF, Cayla G, de La Coussaye JE (2011) Les filières de soins au cours du syndrome coronarien aigu avec susdécalage permanent du segment ST. Ann Fr Med Urg 1(Suppl 2): (in press)

3. Charpentier S, Lauque D (2011) Douleur thoracique et syndromes coronariens aigus : stratégie diagnostique. Ann Fr Med Urg 1:(in press)

4. Jacquin L, Auffray JP, Bessereau J, et al (2011) Le ticagrelor dans les syndromes coronaires aigus : résultats de l'étude PLATO. Ann Fr Med Urg 1:(in press)

5. Wallentin L, Becker RC, Budaj A, et al (2010) Ticagrelor versus clopidogrel in patients acute coronary syndromes. N Engl J Med 361:1045-57

6. Assez N, Wiel E, Lemanski-Brulin C, et al (2011) Syndromes coronariens aigus : prise en charge thérapeutique en urgence. Ann Fr Med Urg 1:(in press)

7. Villemant D, Barriot P, Riou B, et al (1987) Achievement of thrombolysisat home in cases of acute myocardial infarction. Lancet 1:228-9

8. Weiss AT, Fine DG, Applebaum D, et al (1987) Prehospital coronary thrombolysis. A new strategy in acute myocardial infarction. Chest 92:124-8

9. Montalescot G, Zeymer U, Silvain J, et al (2011) Intravenous enoxaparin or unfractionned heparin in primary percutaneous coronary intervention for ST-elevation myocardial infarction: the international randomized open-label ATOLL trial. Lancet 378:693-703 